\title{
On the use of MHD mode analysis as a technique for determination of q-profiles in JET plasmas
}

\author{
M. F. F. Nave, D. Borba, R. Galvão, and S. Hacquin \\ Centro de Fusão Nuclear Associação Euratom-IST, Instituto Superior Técnico, 1049-001 Lisboa, Portugal
}

B. Alper, C. Challis, S. Gerasimov, N. Hawkes, J. Mailloux, and S. Sharapov

Euratom/UKAEA Fusion Association, Culham Science Centre, Abingdon OX14 3DB, United Kingdom

C. Boswell

Plasma Science and Fusion Center, MIT, Cambridge, Massachusetts 02139

M. Brix

Institut für Plasmaphysik, Forschungszentrum Jülich GmbH, EURATOM Association, TEC, 55428 Jülich, Germany

E. Joffrin

Association EURATOM-CEA sur la Fusion Controlee Cadarache, Saint Paul-lez-Durance, France

E. de la Luna

Asociation EURATOM-CIEMAT para Fusion, CIEMAT, Spain

P. Smeulders

Associazioni EURATOM-ENEA sulla Fusione, C.R. Frascatti, 1-00044, Roma, Italy

EFDA-JET Contributors ${ }^{\text {a) }}$

(Presented on 22 April 2004; published 18 October 2004)

JET plasmas show fluctuations from a large variety of MHD instabilities. A combination of spectral analysis, mode number analysis, and determination of mode radial position has the potential as a diagnostic for the plasma $q$ profile. Mode frequencies and mode numbers are obtained from spectral and phase analysis from fast magnetic pickup signals. Cross correlation of magnetic and multiarray signals from ECE, SXR, and reflectometer systems provide the mode localization. The present status of the diagnostic systems used for fluctuation analysis is summarized, with particular emphasis on the fast magnetic pickup system. A new data collection system allows the magnetic signals to be recorded at $2 \mathrm{MHz}$ for up to $32 \mathrm{~s}$ in each pulse. An example of $q$-profile validation from the observation of Alfven eigenmode cascades and a snake is given. At JET, Alfven cascades are used to determine $q_{\min }$ in optimized shear discharges, while a variety of low frequency modes (such as the snake) allow the determination of the radial position of $q$ integer values in monotonic and reversed shear $q$ profiles. (C) 2004 American Institute of Physics. [DOI: 10.1063/1.1791333]

\section{INTRODUCTION}

MHD mode identification backed by MHD modeling has been used from the early days of JET operation as a diagnostic for the plasma $q$ profile. For instance, observation of external kink modes and double tearing modes helped to understand current penetration during the plasma start up and to design a safe (disruption-free) current ramp up. ${ }^{1,2}$ Another early example is the calibration of the central $q$ value from the observation of sawteeth and the input of constraints (as the sawtooth inversion radius or the radial position of low mode number tearing modes) in the codes IDENTC and IDENTD. ${ }^{3}$ The localization of the $(1,1)$ snake observed after $D_{2}$ pellet injection has provided the evolution of the $q=1$ surface in between sawtooth crashes in plasmas with mono-

${ }^{a}$ See appendix of the paper by J. Pamela in Proceedings of IAEA Conference on Fusion Energy, Sorrento, Italy 2000. tonic $q$ profiles. ${ }^{4}$ More recently, MHD modes have been extensively used to calibrate $q$ profiles obtained with the code EFIT taking into account MSE measurements. ${ }^{5}$ Observation of Alfven cascades has become a standard tool for the determination of the evolution of the minimum $q$ in advanced scenarios with optimised shear. ${ }^{6-10}$

MHD fluctuations on JET are studied with a set of fast Mirnov coils placed at different poloidal and toroidal positions. Helical structures with mode numbers $n \leqslant 10$ and $m$ $\leqslant 20$ can be determined. The radial location of the fluctuations is obtained by the measurement of electron cyclotron emission (ECE) and plasma radiation in the soft x-ray (SXR) wavelength. Finally, MHD modes can be identified from the signal fluctuations of a microwave reflectometry/ interferometry diagnostic. Cross correlation of observations in different diagnostics is possible by synchronous data collection of all the above diagnostic signals using a central acquisition and trigger system (CATS). ${ }^{11}$ The ECE, SXR, and $\mathrm{O}$-mode reflectometer diagnostic measurements allow 


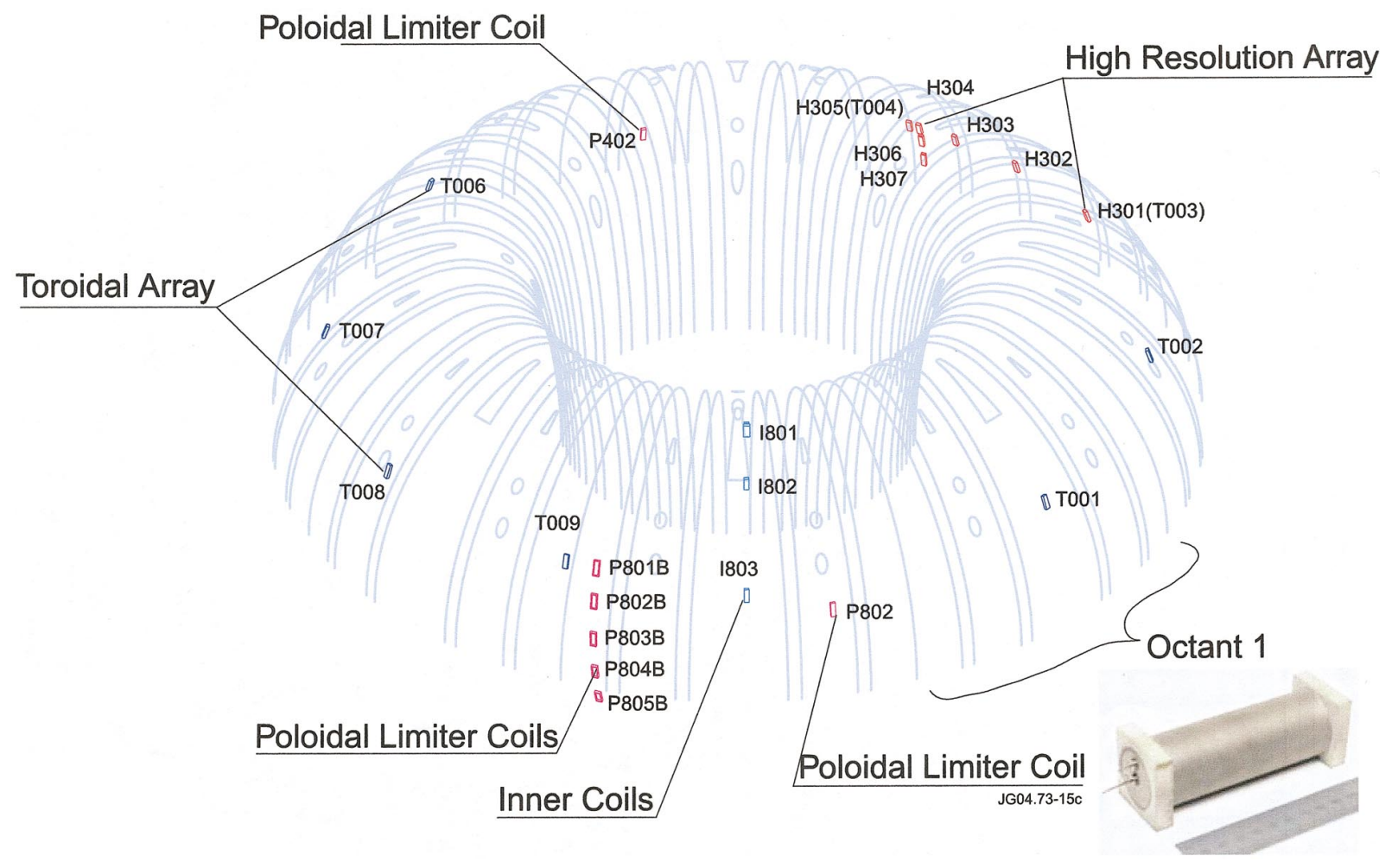

FIG. 1. (Color) Fast magnetic coil layout and an image of a fast magnetic coil.

the identification of low amplitude, core MHD modes not measured by the external magnetic pickup coils.

\section{FAST MAGNETIC PICKUP COILS}

A set of fast magnetic pickup coils was designed for high frequency MHD activity study (up to $500 \mathrm{kHz}$ ). They were wound from titanium wire in a single layer onto a ceramic former with a return wire through the center of the coil. A typical coil diameter of $35 \mathrm{~mm}$ was used with about 70 turns of $0.5 \mathrm{~mm}$ diameter wire with $1.5 \mathrm{~mm}$ pitch. The coil length is about $100 \mathrm{~mm}$. The coils are in the torus vacuum, shielded by individual stainless steel cases with a longitudinal slot. The plasma facing surface of the stainless steel case is protected by a carbon tile case. The butt ends of the coils were not protected either by stainless steel or carbon case.

There are seven "High Resolution Array" coils outboard of the plasma distributed toroidally and poloidally at Octant 3 , which are basically used for high toroidal (and poloidal) mode analysis. Six "Toroidal Array" coils and two "Poloidal Limiter Coils" (at octants 4 and 8) are distributed toroidally around the torus outboard of the plasma, which are used for low MHD toroidal mode analysis. Three "Inner Coils" are inboard of the plasma and five "Poloidal Limiter Coils" outboard of the plasma. Together with the "High Resolution Array" coils, these are used for poloidal mode number determination. (The present coil layout is shown in Fig. 1. For planned upgrades see Ref. 12.)

A new data collection system (named $\mathrm{KC} 1 \mathrm{M}$ has been commissioned in 2004 that allows the magnetic signals to be recorded at $2 \mathrm{MHz}$ for up to $32 \mathrm{~s}$ in each pulse. The system is based on four-channel ADC cards in PCI format, installed in a PC running LINUX. Sampling is started from an exter- nal trigger and synchronized to an external clock, both derived from the JET central timing system. Each ADC channel converts to 14-bit resolution and is electronically isolated from its neighbors. To date, 23 MHD coils have been digitised, together with eight selectable reflectometer channels. The system will be enlarged to 32 magnetic MHD signals and 32 other selectable fast diagnostic signals in 2005.

\section{DIAGNOSTICS USED FOR MHD MODE LOCALIZATION}

\section{A. Soft-x-ray cameras}

The complete mutichannel SXR system ${ }^{13}$ is based on twelve in-vessel 35-element photodiode arrays together with two radiation shielded external cameras each with 17 views. Due to radiation damage to in-vessel detectors, primarily during $D-T$ operation, current MHD analysis relies on one vertically mounted camera of 35 views and one horizontally mounted camera of 17 views (Fig. 2). The system is sensitive to SXRs in the energy range $2-10 \mathrm{keV}$. As well as use in snake, fishbone, and sawtooth studies, cross-correlation analyses with magnetic signals are used to supplement mode localization information and the phase variation across the channels can distinguish even and odd poloidal mode numbers.

\section{B. Microwave O-mode reflectometer/interferometer}

A multichannel O-mode microwave reflectometer system working at fixed frequency is used to measure the density fluctuations in JET. ${ }^{14}$ Information on mode localization is provided from the reflectometry channels whose cutoff frequency is within the plasma (reflectometry mode). In this 

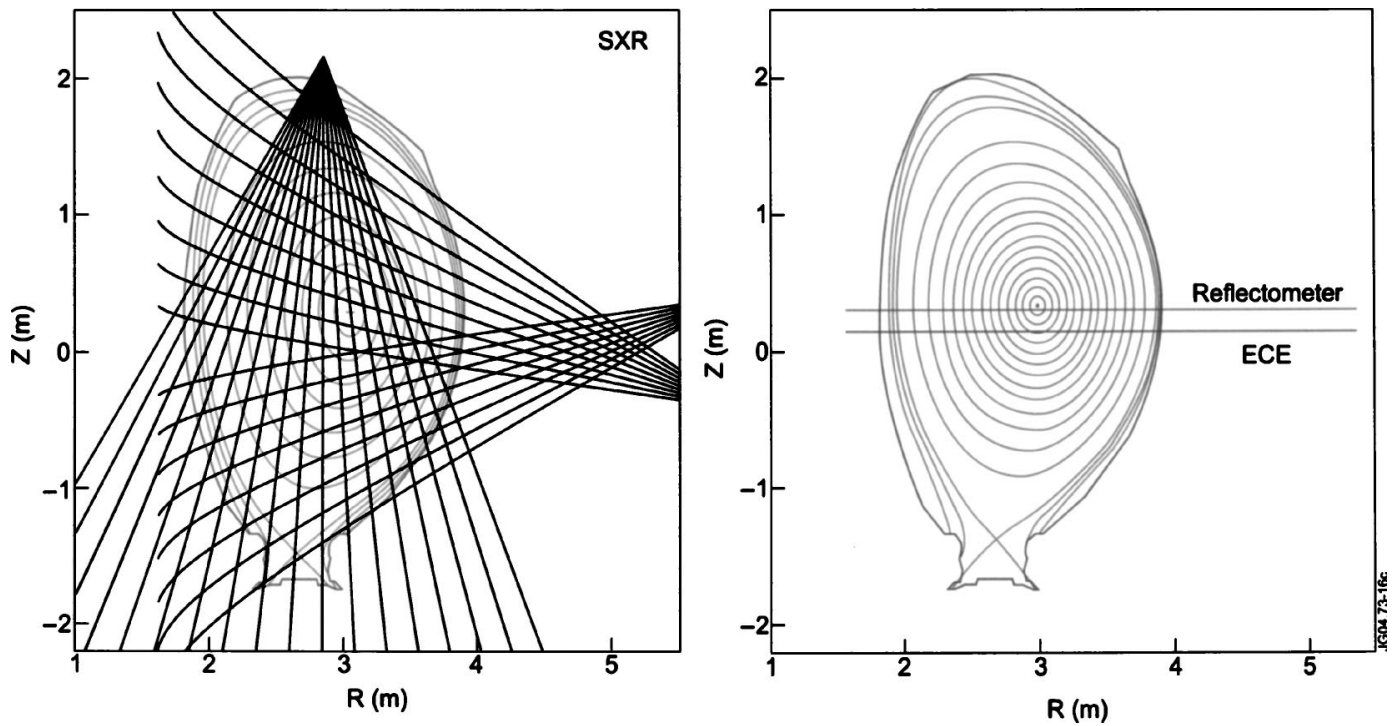

FIG. 2. (a) JET poloidal cross section with SXR vertical (showing 18 of 35 lines of sight) and horizontal (17 lines of sight) camera views; (b) JET poloidal cross section with lines of sight of ECE radiometer and microwave interferometer/reflectometer.

mode probing waves are reflected at different plasma densities providing a spatial localization of the density fluctuations. When the plasma density everywhere is low enough such that there is no cutoff frequency, the system acts as an interferometer with the probing waves reflecting off the inner wall. In this mode (interferometry mode) the reflectometer gives a line-averaged measurements of the density fluctuations.

\section{Electron cyclotron emission}

An ECE heterodyne radiometer with an array of 48 (recently upgrade to 96) channels is used for electron temperature measurements. ${ }^{15}$ It has a special resolution of $1 \mathrm{~cm}$. In most plasmas, the ECE diagnostic line of sight lies below the plasma midplane (Fig. 2). The angular separation between the positions of two channels with respect to the magnetic axis can be used to determine the poloidal mode number. This is useful in the case of low amplitude oscillations not seen with the magnetic pickup coils. Also, it can give a local determination of the poloidal mode number of core oscillations such as sawtooth precursors, whereas the magnetic data give a larger $m$ due to toroidal coupling.

\section{EXAMPLE OF MHD ANALYSIS AND $q$ PROFILE VALIDATION}

As an example of the potential use of mode analysis for $q$-profile calibration we consider an optimized shear discharge from a recent trace Tritium JET campaign. A spectrogram (Fig. 3) from a microwave signal in interferometry mode shows Alfven Eigenmode (AE) cascades with frequencies ranging from 100 to $250 \mathrm{kHz}$, with toroidal mode numbers $n \geqslant 5$. At lower frequencies $(5-50 \mathrm{kHz})$ snakes are observed. We consider here the snake observed at $t=5.4 \mathrm{~s}$. The snake first harmonic has mode numbers $m=3, n=1$. ECE data show that they are localized at $3.5 \mathrm{~m}$ (Fig. 4).

Measurements of AE instabilities are used to solve the inverse problem of identifying the plasma parameters. In the case of reversed shear plasmas the Alfvén Cascades are used to determine the minimum of the safety factor $\left(q_{\min }\right)$ as shown in Fig. 3. The frequency pattern of the AE cascades reflects the time evolution of the Alfvén continuum at the location of $q_{\min }$ which is linked to the frequency of the eigenmode located at that surface. ${ }^{6-9}$ The AE Cascade frequency, being related to the Alfvén continuum frequency at $q=q_{\text {min }}$, is given by

$$
\omega=\left|\frac{m}{q_{\min }}-n\right| \frac{V_{A}}{R_{0}}+n \omega_{\mathrm{rot}}+\Delta \omega,
$$

where $\omega$ is the observed frequency, $m$ the poloidal mode number, $n$ the toroidal mode number, $R_{0}$ major radius, $V_{A}$ the Alfvén velocity, $\omega_{\text {rot }}$ is the rotation frequency of the plasma and $\Delta \omega$ is a frequency offset. ${ }^{7}$ The presence of eigenmodes with different $n$ and $m$ yields the periodic pattern of sweeping frequencies which characterizes the AE cascades. Mea-
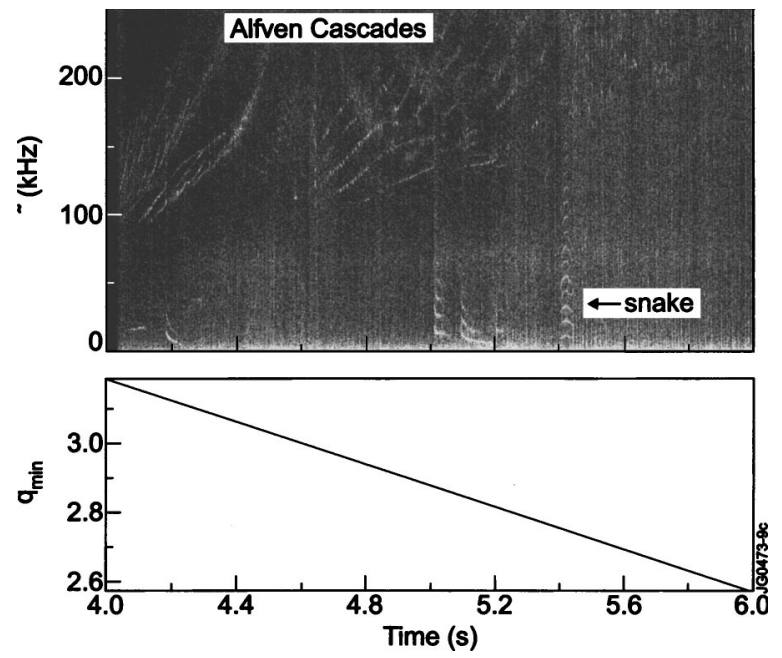

FIG. 3. (a) Spectrogram from microwave interferometer signal showing AE modes and snake-like modes; (b) estimated $q_{\text {min }}$ from AE cascades with toroidal mode numbers $n=5-15$, assuming a constant plasma rotational frequency of $10 \mathrm{kHz}$. 

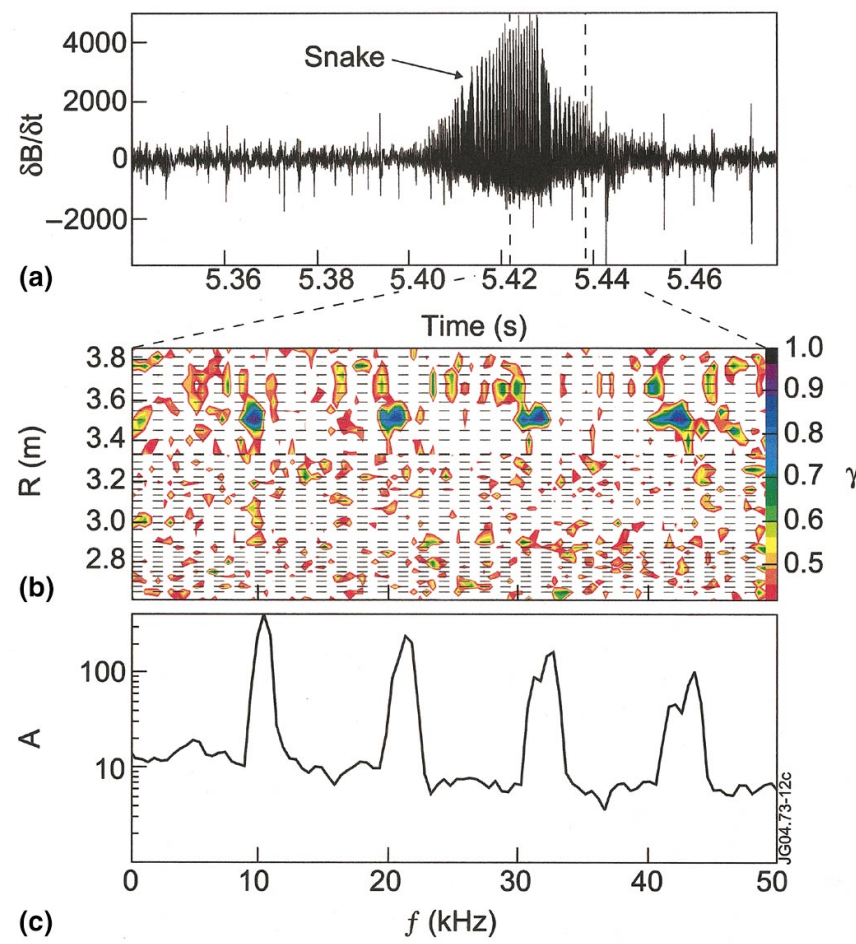

FIG. 4. (Color) (a) Magnetic pickup signal showing snake burst; (b) ECE coherence with magnetic signal; (c) magnetic signal power spectrum. The snake is localized at $3.5 \mathrm{~m}$ that is at the base of the internal transport barrier.

surements of the Alfvén instabilities performed using the reflectometer diagnostic are able to detect eigenmodes with higher toroidal mode numbers, ${ }^{16}$ allowing more accurate determination of the $\left(q_{\min }\right)$ evolution as shown in Fig. 4(b).

At the time the snake is observed, the $q$ profile is expected to have a noninterger minimum $q, q_{\min } \sim 2.7-2.8$ (from $\mathrm{AE}$ analysis) and a $q=3$ value at $3.5 \mathrm{~m}$. Figure 5 shows EFIT reconstructions of the $q$-profile with different fitting constraints at the time of the snake. Only the MSE data $^{5}$ detects the current hole, however, if the detailed features of the MSE measurements are too closely fitted by the equilibrium code then large overshoots in the $q$-profile can occur. The $q$ profile for the closely fitted MSE data (curve 3) results in a significantly smaller $q_{\min }$ than is indicated by the MHD events. The MHD analysis is an important guide in selecting the optimum curvature of the fitted profiles.

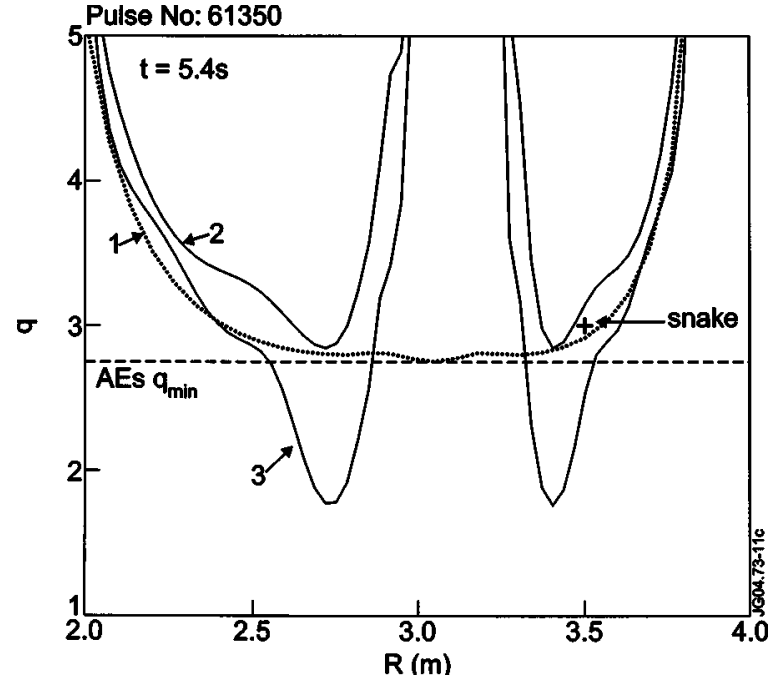

FIG. 5. $q$ profile from EFIT with different boundary conditions: (1) magnetics only; (2) and (3) with magnetics and MSE data fitting MSE data approximately (2) or very closely (3). Indicated in the figure is the estimated $q_{\min }$ from $\mathrm{AE}$ cascade analysis and the radius of $q=3$ obtained from the snake.

\section{ACKNOWLEDGMENTS}

This work was performed under the European Fusion Development Agreement. It received financial support from Fundação para a Ciencia e Tecnology (FCT), Portugal. The content of the publication is the sole responsibility of the authors and it does not necessarily represent the views of the Commission of the European Union or FCT or their services.

${ }^{1}$ P. R. Thomas et al., Proceedings of the Workshop Tokamak Start-Up, Erice, 14-20 July 1985.

${ }^{2}$ D. J. Campbell et al., Nucl. Fusion 28, 981 (1988).

${ }^{3}$ J. Blum et al., Nucl. Fusion 30, 1475 (1990).

${ }^{4}$ R. D. Gill et al., Nucl. Fusion 32, 723 (1992).

${ }^{5}$ B. C. Stratton, D. Long, R. Palladino, and N. C. Hawkes, Rev. Sci. Instrum. 70, 898 (1999).

${ }^{6}$ H. Berk et al., Phys. Rev. Lett. 87, 185002 (2001).

${ }^{7}$ S. E. Sharapov et al., Phys. Lett. A 289, 127 (2001).

${ }^{8}$ D. Borba et al., Nucl. Fusion 42, 1029 (2002).

${ }^{9}$ A. Fasoli et al., Plasma Phys. Controlled Fusion 44B, 159 (2002).

${ }^{10}$ J. Mailloux et al., Phys. Plasmas 9, 2156 (2002).

${ }^{11}$ K. Blackler and A. W. Edwards, IEEE Trans. Nucl. Sci. 41, 111 (1994).

${ }^{12}$ V. Coccorese, Rev. Sci. Instrum., these proceedings.

${ }^{13}$ B. Alper et al., Rev. Sci. Instrum. 68, 778 (1997).

${ }^{14}$ A. Sips and G. Kramer, Plasma Phys. Controlled Fusion 35, 743 (1993).

${ }^{15}$ E. de la Luna, Rev. Sci. Instrum., these proceedings.

${ }^{16}$ S. E. Sharapov et al., Phys. Rev. Lett. (submitted). 